\title{
Hot accretion flow with anisotropic viscosity
}

\author{
Mao-Chun $\mathrm{Wu}^{1}$, De-Fu Bu${ }^{1}$, Zhao-Ming Gan ${ }^{1}$, and Ye-Fei Yuan ${ }^{2}$ \\ 1 Key Laboratory for Research in Galaxies and Cosmology, Shanghai Astronomical Observatory, Chinese Academy of Sciences, \\ 80 Nandan Road, 200030 Shanghai, PR China \\ e-mail: [mcwu;dfbu]@shao.ac.cn \\ 2 Key Laboratory for Research in Galaxies and Cosmology, Department of Astronomy, \\ University of Science and Technology of China, Hefei, 230026 Anhui, PR China
}

Received 17 March 2017 / Accepted 16 September 2017

\begin{abstract}
In extremely low accretion rate systems, the ion mean-free path can be much larger than the gyroradius. Therefore, gas pressure is anisotropic with respect to magnetic field lines. The effects of pressure anisotropy can be modeled by an anisotropic viscosity with respect to magnetic field lines. Angular momentum can be transferred by anisotropic viscosity. In this paper, we investigate hot accretion flow with anisotropic viscosity. We consider the case that anisotropic viscous stress is much larger than Maxwell stress. We find that the flow is convectively unstable. We also find that the mass inflow rate decreases towards a black hole. Wind is very weak; its mass flux is $10-15 \%$ of the mass inflow rate. The inward decrease of inflow rate is mainly due to convective motions. This result may be useful to understand the accretion flow in the Galactic Center Sgr A* and M 87 galaxy.
\end{abstract}

Key words. accretion, accretion disks - black hole physics - hydrodynamics - ISM: jets and outflows - conduction

\section{Introduction}

Hot accretion flows, such as advection-dominated accretion flows (ADAFs; Narayan \& Yi 1994, 1995; Abramowicz et al. 1995) are of great interest because they are likely operating in low-luminosity active galactic nuclei (AGN), that is, the majority of galaxies at least in the nearby Universe, hard and quiescent states of black hole X-ray binaries (see Yuan \& Narayan 2014, for the latest review). Many numerical simulations have been carried out to study the structure of hot accretion flows (e.g., Igumenshchev \& Abramowicz 1999, 2000; Stone et al. 1999; Stone \& Pringle 2001; Machida et al. 2001; Hawley \& Balbus 2002; Pang et al. 2001; Yuan et al. 2012a).

One of the most important findings by numerical simulations in this field is the discovery of strong wind launched from the accretion flow (Yuan et al. 2012b; Narayan et al. 2012; Li et al. 2013; Sadowski et al. 2016). This result was soon confirmed by the 3 million seconds of Chandra observation of the accretion flow around the super massive black hole in the Galactic Center, combined with modeling of the detected iron emission lines (Wang et al. 2013). Begelman (2012) and Gu (2015) analytically address the reason for the existence of wind in hot accretion flows.

In extremely-low-accretion-rate hot accretion flow, such as the accretion flow in the Galactic Center, Sgr A*, and M 87, the Coulomb mean-free path of both ions and electrons is much larger than the typical length-scale of the accretion flow, $\sim G M / c^{2}$ (Mahadevan \& Quataert 1997; Foucart et al. 2016), where $M \equiv$ black hole mass, $G \equiv$ gravitational constant, and $c \equiv$ speed of light. At first glance, the plasma is collisionless. However, particle-in-cell simulations of shear flows have shown that the effective collision rate of particles can be increased by wave-particle interactions (Kunz et al. 2014; Hellinger \& Trávníček 2015; Riquelme et al. 2015; Sironi \& Narayan 2015). This conclusion is supported by measurements in the solar wind, which show that the gas is not totally collisionless (e.g. Kasper et al. 2002; Hellinger et al. 2006; Kulsrud 2004). This is what we shall call the weakly collisional accretion flow. In weakly collisional accretion flow, non-ideal processes such as conduction and pressure anisotropy are likely to be important (Chandra et al. 2015; Foucart et al. 2016).

Previous works (Tanaka \& Menou 2006; Johnson \& Quataert 2007; Quataert 2008; Parrish \& Stone 2005, 2007; Sharma et al. 2008; Bu et al. 2011, 2016; Foucart et al. 2016) have shown that thermal conduction can affect the dynamics of accretion flow significantly. Thermal conduction can transport energy from the inner (hotter) to the outer (cooler) regions. If the energy flux carried by thermal conduction is substantial, the temperature of the gas in the outer regions can be increased above the virial temperature. Thus, gas in the outer regions is able to escape from the gravitational potential of the central black hole and form outflows, significantly decreasing the mass accretion rate. Bu et al. (2016) studied the effects of thermal conduction on wind properties and found that, in the presence of conduction, the energy flux carried by wind (or outflow) can be increased by a factor of $\sim 10$.

In weakly collisional accretion flow, pressure parallel to magnetic field is different from that perpendicular to magnetic field (Chandra et al. 2015). Previous works show that anisotropic pressure can affect the properties of accretion flow significantly. For example, if the pressure is anisotropic, the growth rate of magneto-rotational instability (MRI; Balbus \& Hawley 1991, 1998) can be increased (Quataert et al. 2002; Sharma et al. 2003).

Recently, studies show that the effects of pressure anisotropy can be modeled by an anisotropic viscosity with respect to magnetic field lines (Balbus 2004; Islam \& Balbus 2005; Islam 2014; Chandra et al. 2015; Foucart et al. 2016). Therefore, in weakly collisional accretion flow, even without viscous stress induced by MRI, the angular momentum of gas can be transferred. The 
purpose of this paper is to study weakly collisional hot accretion flow. We mainly focus on accretion flow in which anisotropic viscous stress is much larger than Maxwell stress. Especially, we pay attention to a question that whether wind can be produced. We are interested in wind production because wind is not only an important ingredient of accretion physics but also plays an important role in AGN feedback (Ostriker et al. 2010).

In Sect. 2, we present the basic equations and the simulation models. In Sect. 3, we present the main results. We discuss and summarize our results in Sect. 4.

\section{Numerical method and models}

\subsection{Equations}

In spherical coordinates $(r, \theta, \phi)$, we solve the following magneto-hydrodynamic (MHD) equations using ZEUS-2D code (Stone \& Norman 1992a,b):

$\frac{\mathrm{d} \rho}{\mathrm{d} t}+\rho \nabla \cdot \boldsymbol{v}=0$

$\rho \frac{\mathrm{d} v}{\mathrm{~d} t}=-\nabla p-\rho \nabla \Phi+\frac{1}{4 \pi}(\nabla \times \boldsymbol{B}) \times \boldsymbol{B}+\nabla \cdot \boldsymbol{\Pi}$,

$\rho \frac{\mathrm{d}(e / \rho)}{\mathrm{d} t}=-p \nabla \cdot \boldsymbol{v}+\boldsymbol{\Pi} \nabla \cdot \boldsymbol{v}+\eta \boldsymbol{J}^{2}$,

$\frac{\partial \boldsymbol{B}}{\partial t}=\nabla \times(\boldsymbol{v} \times \boldsymbol{B}-\eta \boldsymbol{J})$

where $\rho$ is density, $\boldsymbol{v}$ is velocity, $p$ is gas pressure, $e$ is gas internal energy, $\boldsymbol{B}$ is magnetic field, $\boldsymbol{J}=(\boldsymbol{c} / 4 \pi \nabla \times \boldsymbol{B})$ is current density, and $\Phi$ is gravitational potential. We use pseudo-Newtonian potential (Paczyński \& Wiita 1980) to mimic the general relativistic effects, $\Phi=-\mathrm{GM} /\left(r-R_{\mathrm{s}}\right)$, where $R_{\mathrm{s}}=2 \mathrm{GM} / c^{2}$ is the Schwarzschild radius. We adopt an adiabatic equation of state $p=(\gamma-1) e$, and set $\gamma=5 / 3$.

In Eqs. (2) and (3), we use anisotropic viscous stress tensor $\mathbf{I}$ to model the effects of ion pressure anisotropy (Braginskii 1965):

$\boldsymbol{\Pi}=-3 \rho v\left[\hat{b} \hat{b}: \nabla \boldsymbol{v}-\frac{\nabla \cdot \boldsymbol{v}}{3}\right]\left[\hat{b} \hat{b}-\frac{\boldsymbol{I}}{3}\right]$,

where $\hat{\boldsymbol{b}}=\boldsymbol{B} /|\boldsymbol{B}|$ is a unit vector in the direction of magnetic field, and $\boldsymbol{I}$ is the unit tensor. The viscous stress can be written as

$\Delta \boldsymbol{P}=-3 \rho v\left[\hat{b} \hat{b}: \nabla \mathbf{v}-\frac{\nabla \cdot \boldsymbol{v}}{\mathbf{3}}\right]=P_{\|}-P_{\perp}$,

where $P_{\|}=P+\Delta P_{\|}$is pressure along magnetic field line and $P_{\perp}=P+\Delta P_{\perp}$ is pressure perpendicular to magnetic field line, in which $\Delta P_{\|}$and $\Delta P_{\perp}$ are deviations from $P$ in the directions along and perpendicular to magnetic field, respectively. $\Delta P_{\|}$and $\Delta P_{\perp}$ vary with time and give rise to a shear viscosity and a bulk viscosity, respectively. Chandra et al. (2015) show that there is a relation between $\Delta P_{\|}$and $\Delta P_{\perp}$. The relation is that $\Delta P_{\|}=-2 \Delta P_{\perp}$, $\Delta P_{\|}$and $\Delta P_{\perp}$ are comparable. The magnitude of shear viscosity and bulk viscosity is comparable, so we use the same coefficient for both shear and bulk viscosity. In Eq. (6), $v$ is viscous coefficient, we adopt $v=\phi c_{\mathrm{s}}^{2} \tau_{\mathrm{R}}$ (Foucart et al. 2016), $\phi$ is a constant, $c_{\mathrm{S}}$ is sound speed, and $\tau_{\mathrm{R}}$ is effective ion mean-free-time between Coulomb scatterings, which is roughly equal to the orbital time of the accretion flow. In this paper, we take $v=\phi r^{1 / 2}$ (by assuming the gas has virial temperature, $\left.c_{\mathrm{s}}^{2}=\mathrm{GM} / r, \tau_{\mathrm{R}} \approx \sqrt{r^{3} /(\mathrm{GM})}\right)$ as our fiducial diffusion coefficient.
Table 1. Simulation parameters.

\begin{tabular}{ccccc}
\hline \hline Models & Field topology & $\beta_{0}$ & $\phi$ & $t_{\mathrm{f}}{ }^{\star}$ \\
\hline A & dipole & $10^{4}$ & 0.02 & 25 \\
$\boldsymbol{B}$ & dipole & $10^{8}$ & 0.02 & 25 \\
$\mathrm{C}$ & dipole & $10^{8}$ & 0.01 & 50 \\
$\mathrm{D}$ & quadrupole & $10^{8}$ & 0.01 & 25 \\
$\mathrm{E}$ & dipole & 200 & 0.02 & 10 \\
\hline
\end{tabular}

Notes. ${ }^{(\star)}$ Final time at which each simulation is stopped and time is in units of the orbital time at $R_{0}=100 R_{\mathrm{s}}$.

The final terms in Eqs. (3) and (4) are the magnetic heating and dissipation rate mediated by a finite resistivity $\eta$. Since the energy equation here is actually an internal energy equation, numerical reconnection inevitably results in loss of energy from the system. By adding the anomalous resistivity, the energy loss can be captured in the form of heating in the current sheet. The exact form of $\eta$ is the same as that used by Stone \& Pringle (2001). The effects of this non-ideal magnetic field treatment are described in the Appendix A.

\subsection{Initial conditions and numerical method}

The initial condition is an equilibrium torus with constant specific angular momentum given by Stone \& Pringle (2001). In this paper, we assume $G=M=1$. The initial torus center is located at $R_{0}=100 R_{\mathrm{s}}$. Time is expressed in units of the orbital time at $100 R_{\mathrm{s}}$.

The computational domain is from $R_{\text {in }}=1.35$ to $R_{\text {out }}=$ $400 R_{\mathrm{S}}$ in the radial direction and from $\theta=0$ to $\theta=\pi$ in the angular direction. We use logarithmically spaced grids in the radial direction. In the angular direction, the grids are uniformly spaced. The axisymmetric boundary conditions are used at $\theta=0$ and $\theta=\pi$. Outflow boundary conditions are adopted at both the inner and outer radial boundaries. Our numerical resolution is $168 \times 88$

\subsection{Models}

The magnetic field which threads the torus initially is generated by a vector potential, that is, $\boldsymbol{B}=\nabla \times \boldsymbol{A}$. Initializing the magnetic field in this way guarantees that it will be divergence free. In this work, we take $\boldsymbol{A}$ to be purely azimuthal. We consider two magnetic field configurations: dipolar $\boldsymbol{A}_{\phi}=\rho^{2} / \beta_{0}$ and quadrapolar $\boldsymbol{A}_{\phi}=r \cos (\theta) \rho^{2} / \beta_{0} . \beta_{0}=P_{\text {gas }} / P_{\text {mag }}$.

Table 1 lists the main parameters in all models presented here, initial magnetic field topology, initial plasma parameter $\beta_{0}$, viscous coefficient $\phi$, and final time $t_{\mathrm{f}}$ at which each simulation is stopped (all times in this paper are in units of the orbital time at $R_{0}=100 R_{\mathrm{s}}$ ).

\section{Results}

We analyze the properties of hot accretion flow at the quasisteady state, that is, the net accretion rate is independent of radius. The angle integrated mass inflow and outflow rates, $\dot{M}_{\text {in }}$ and $\dot{M}_{\text {out }}$, are defined as follows,

$$
\begin{aligned}
& \dot{M}_{\mathrm{in}}(r)=2 \pi r^{2} \int_{0}^{\pi} \rho \min \left(v_{r}, 0\right) \sin \theta \mathrm{d} \theta \\
& \dot{M}_{\mathrm{out}}(r)=2 \pi r^{2} \int_{0}^{\pi} \rho \max \left(v_{r}, 0\right) \sin \theta \mathrm{d} \theta
\end{aligned}
$$




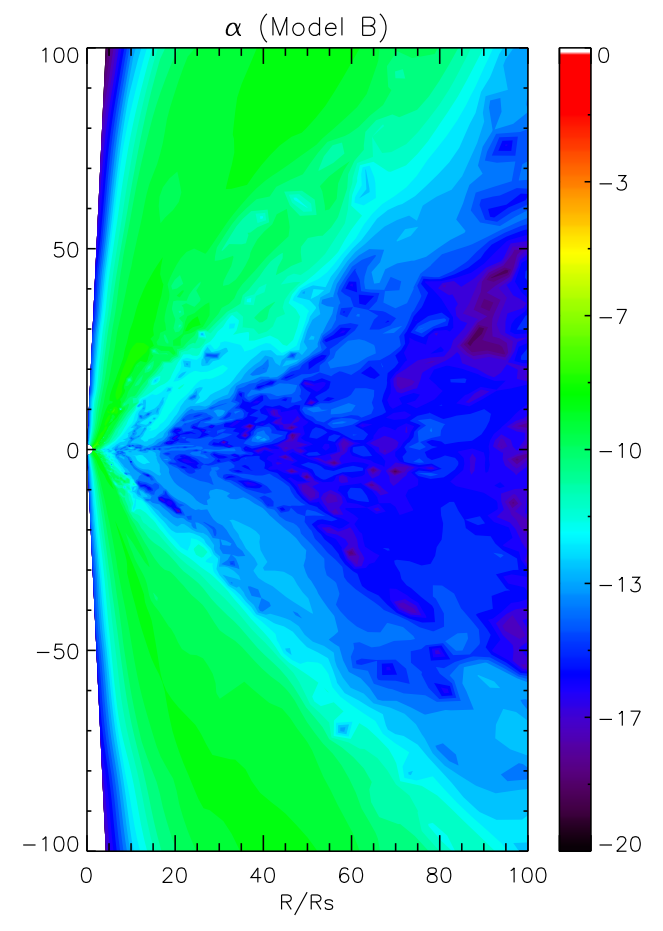

Fig. 1. Time-averaged (from $t=20$ to 21.5 orbits) logarithm $\alpha=$ $-B_{r} B_{\phi} / 4 \pi p$ for Run B.

and the net mass accretion rate is,

$\dot{M}_{\text {acc }}(r)=\dot{M}_{\text {in }}(r)+\dot{M}_{\text {out }}(r)$.

We note that the above rates are obtained by time-averaging the integrals rather than integrating the time averages.

\subsection{A fiducial model - Run B}

Model B $\left(\beta_{0}=10^{8}, \phi=0.02\right)$ is chosen as our fiducial model. In model $\mathrm{B}$, the initial magnetic field is very weak. When quasisteady state is achieved, the Maxwell stress is much weaker than the anisotropic viscosity. We define the viscous coefficient induced by Maxwell stress as $\alpha=-B_{r} B_{\phi} / 4 \pi p$.

Figure 1 shows the time-averaged $\log \alpha$ for Run B. From this figure, it is clear that in the whole computational domain, $\alpha$ is much smaller than the anisotropic viscosity coefficient $(\phi=$ 0.02 ). Therefore, in this model, angular momentum transfer is dominated by anisotropic viscosity.

The time-averaged mass fluxes of model B are shown in Fig. 2 (black lines). The time-averaged net accretion rate is independent of radius which indicates the flow has reached a quasisteady state. Both the inflow and outflow rates decrease inwards, consistent with those found in previous works (e.g. Stone \& Pringle 2001; Yuan et al. 2012a, 2015). We need to note that in previous works (e.g. Stone \& Pringle 2001; Yuan et al. 2012a, 2015), the angular momentum transfer is induced by Maxwell stress. The inward decrease of mass inflow rate in those works is due to mass loss via real outflow (wind). This is not the case in which Maxwell stress is negligibly small. Below we show that in the case that Maxwell stress is small, mass inflow decreases inward because the accretion flow is convective.

Figure 2 shows that there is a significant mass outflow rate, but this does not mean it is real outflow (wind). The reasons are as follows. According to Eq. (8), when we calculate the mass outflow rate, we include all the gas which has a positive radial

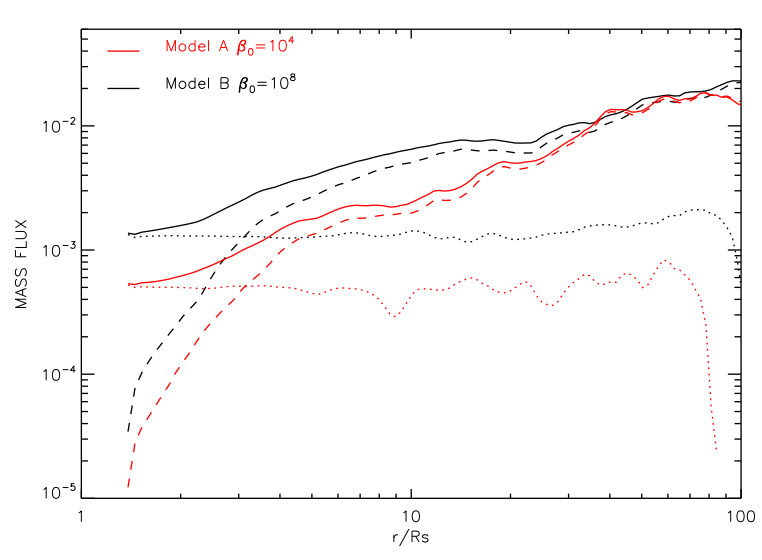

Fig. 2. Radial profiles of the time-averaged (from $t=20$ to 21.5 orbits) and angle integrated mass accretion rate. Solid lines denote the mass inflow rate $\dot{M}_{\text {in }}$ (Eq. (7)), dotted lines denote the net mass accretion $\dot{M}_{\text {net }}$ (Eq. (9)), and dashed lines denote the mass outflow rate $\dot{M}_{\text {out }}$ (Eq. (8)).

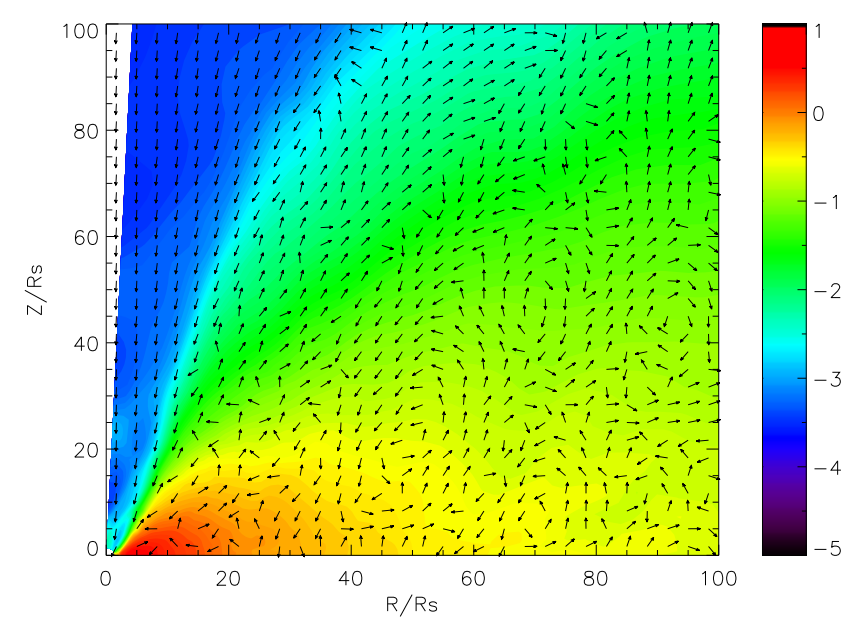

Fig. 3. Time-averaged (from $t=20$ to 21.5 orbits) velocity vectors (arrows) over-plotted by density (colors) for Run B.

velocity. The accretion flow is turbulent. In a turbulent accretion flow, there are real outflow (wind) and turbulent outflow. Real outflow means that the flows are systematically outward moving gas. Turbulent outflow means that the outflow is not real outflow but an outward moving portion of a turbulent eddy.

What is the reason for the inward decrease of mass inflow rate? Does wind exist? To study whether or not wind exists, let us first directly look at the velocity field shown in Fig. 3. In the region $45^{\circ} \leq \theta \leq 90^{\circ}$, turbulent motion dominates. In the region close to the rotational axis, gas is inflowing. It is different from previous simulation results. Previous works show that if the angular momentum is transferred by Maxwell stress (magnetic field is strong), the region close to the rotational axis is filled by jet (e.g., Sadowski et al. 2016; Narayan et al. 2012). In the region $\theta \sim 32^{\circ}$, there is outward moving gas; this portion of outward moving gas returns and becomes inflow at $r \sim 100 R_{\mathrm{s}}$. From Fig. 3, it is hard to find real outflow (wind).

Following Yuan et al. (2015), we now use a much more precise trajectory method to study whether or not winds exist. The details of this approach can be found in Yuan et al. (2015), here we only briefly introduce it. Trajectory is obtained by connecting the positions of the same "test particle" at different times. This concept is related with the Lagrangian description of fluid. We note that this is different from the streamline which is obtained 


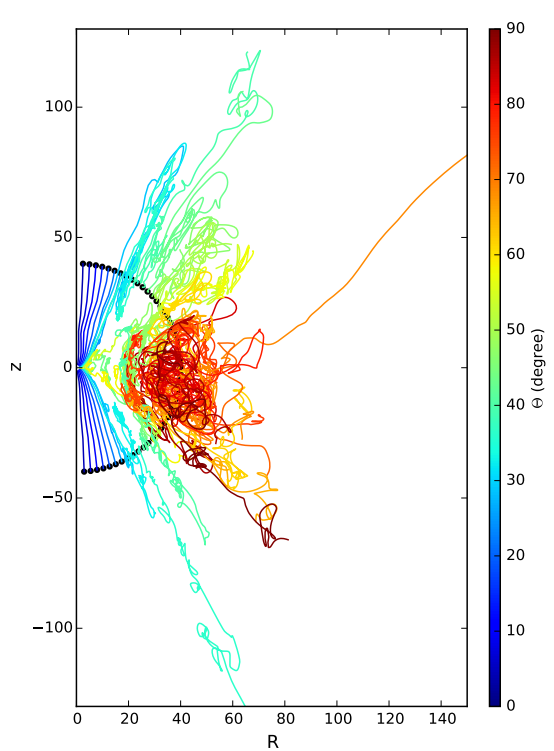

Fig. 4. Trajectories of gas for Run B. The black dots located at $40 R_{\mathrm{S}}$ are starting points of the "test particles". Different colors denote the trajectory of "test particles" starting from different $\theta$ angle. From this Figure we can see that the real wind trajectories, that is, the trajectories which extend from $r=40 R_{\mathrm{S}}$ to large radius and never come back to cross $40 R_{\mathrm{s}}$ are very few. Winds are very weak.

by connecting the velocity vector of different test particles with infinitely short distance at a given time. This concept is associated with the Euler description of fluid motion. Trajectory is only equivalent to the streamline for strictly steady motion, which is not the case for accretion flow since it is always turbulent. To get the trajectory, we first need to choose a virtual "test particle" in the simulation domain. They are of course not real particles, but some grids representing fluid elements. Their locations and velocity at a certain time $t$ are obtained directly from the simulation data. We can then obtain their location at time $t+\delta t$ from the velocity vector and $\delta t$. We do this work using software called "VISIT".

Using a trajectory approach, we can discriminate easily between particles that are real outflows (i.e., winds) and those that are undergoing turbulent motions. We can also calculate the mass fluxes of wind by combining this with the information of density and velocity of wind. For details, see Yuan et al. (2015). Figure 4 shows the trajectories of 25 "test particles" starting from $40 R_{\mathrm{S}}$ in model B. From this figure, we can see that the real wind trajectories, that is, the trajectories that extend from $40 R_{\mathrm{s}}$ to large radius and never cross $40 R_{\mathrm{s}}$ twice, are very few. This implies that the mass flux of wind is very small. Our quantitative calculation confirms this result. For example, we find that, at $40 R_{\mathrm{s}}$, the ratio of mass flux of real outflows to the total outflow rate calculated by Eq. (8) is only $10 \%$. This result means that the mass flux of wind is very small. As a comparison, previous work shows that when the angular momentum transfer is dominated by Maxwell stress (magnetic field is strong), this ratio is $\sim 60 \%$ (Yuan et al. 2015).

Wind is very weak, so what is the reason for the inward decrease of mass inflow rate? According to the above analysis, we find that the mass outflow rate calculated by Eq. (8) is dominated by turbulent outflow. The inward decrease of inflow rate is mainly due to turbulent motions. This reminds us of the scenario of convection-dominated accretion flow (CDAF) proposed by Narayan et al. (2000) and Quataert \& Gruzinov (2000). In the CDAF model, the inward decrease of mass inflow rate is

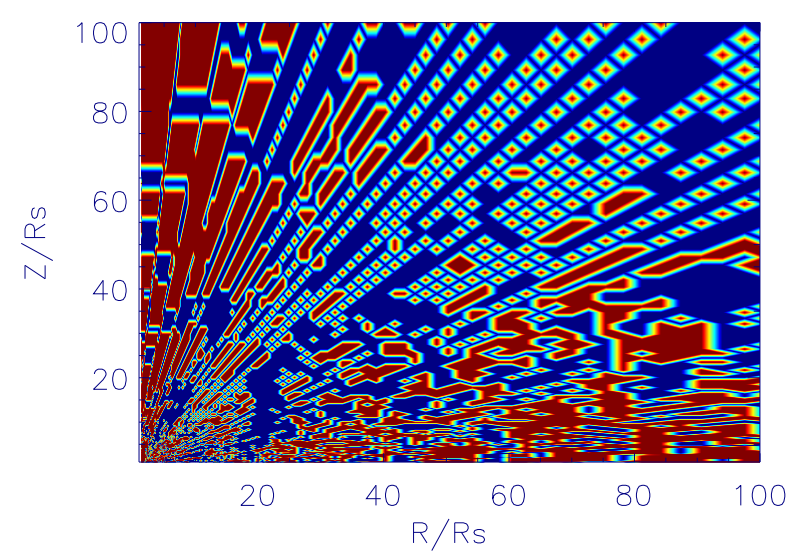

Fig. 5. Time-averaged (from $t=20$ to 21.5 orbits) convective stability analysis for Run B. The red regions are convectively unstable.

induced by turbulent motions. When gas falls inward from larger to smaller radii, more and more gas is locked in turbulent eddies.

We now check whether the accretion flow here is convectively unstable by the Hoiland criteria. (e.g., Tassoul 1978; Begelman \& Meier 1982):

$(\nabla s \cdot \mathrm{d} \boldsymbol{r})(\boldsymbol{g} \cdot \mathrm{d} \boldsymbol{r})-\frac{2 \gamma v_{\phi}}{R^{2}}\left[\nabla\left(v_{\phi} R\right) \cdot \mathrm{d} \boldsymbol{r}\right] \mathrm{d} R<0$.

In Eq. (10), $\mathrm{d} \boldsymbol{r}=\mathrm{d} r \hat{r}+r \mathrm{~d} \theta \hat{\theta}$ is the displacement vector, $s=$ $\ln (p)-\gamma \ln (\rho)$ is the entropy times $(\gamma-1), \boldsymbol{g}=-\nabla \psi+\hat{R} v_{\phi}^{2} / R$ is the effective gravity, and $v_{\phi}$ is the rotational velocity. This condition is equivalent to an inward increase of entropy for non-rotating flow, which is the Schwarzschild criteria. Figure 5 shows the results for Run B. The red regions are convectively unstable. It is clear that the accretion flow is mainly convectively unstable. The physical reason for the instability is that, during the accretion process, heating generated by anisotropic viscosity produces entropy, while the loss of entropy by radiative cooling is neglected. We conclude that our model Run B is very similar to the CDAF model, that is, the turbulent motion driven by convection induces the inward decrease of mass inflow rate.

\subsection{Dependence on the strength of the anisotropic viscosity}

We performed a simulation with smaller viscous coefficient in Run C. The viscous stress in Run C was half of that in the fiducial Run B. With a weaker viscous stress, the evolution of torus proceeds much slowly. Therefore, it takes roughly 40 orbits for the torus to achieve a quasi-steady state. In model $\mathrm{C}$, the Maxwell stress is also much smaller than the anisotropic viscosity. We find that when quasi-steady state is achieved, the mass inflow rate also decreases inwards. The reason for the inward decrease of mass inflow rate is mainly due to turbulent motions induced by convection as is the case found in Run B.

\subsection{Dependence on the initial magnetic field topology}

In order to study whether or not the results depend on the initial magnetic field configuration, we run model D. In this model, the initial magnetic field is quadrapolar. As in models B and $\mathrm{C}$, we find that when the Maxwell stress is much smaller than the anisotropic viscosity, the mass inflow rate decreases inwards. The inward decrease of inflow rate is again due to turbulent motions induced by convection.

Beckwith \& Hawley (2008) studied the effects of magnetic field topology on the evolution of black hole accretion flow. 


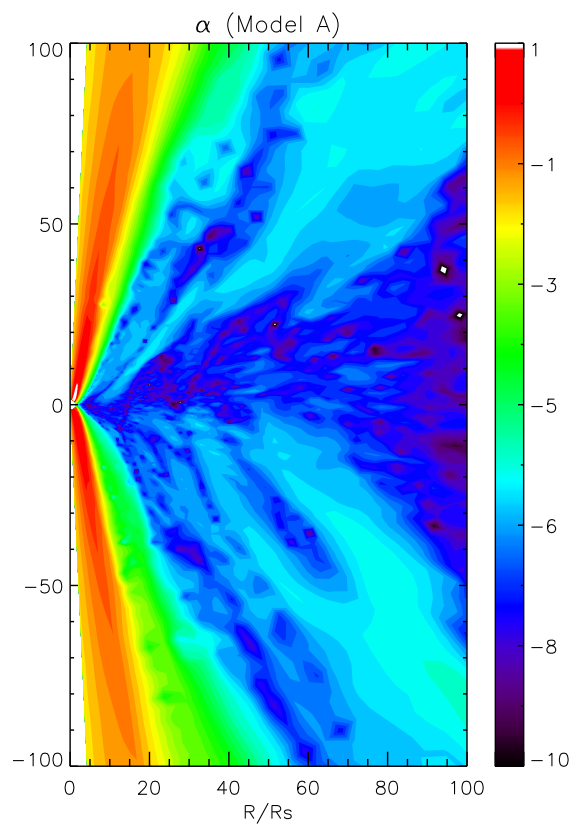

Fig. 6. Same as Fig. 1, but for Run A.

Their results show that the qualitative properties of the accretion flow are independent of initial magnetic field configuration, but jet launching is sensitive to it, that is, a dipolar topology field model has stronger jets than a quadrupolar field model. In the case of extremely weak magnetic field $\left(\beta=10^{8}\right)$, there is no jet (see Fig. 3). Therefore, we can conclude that the properties of accretion flow are independent of initial magnetic field topology when the magnetic field is extremely weak.

\subsection{Dependence on magnetic field strength}

In order to study the dependence on the magnetic field strength, we run models $A$ and $E$. The difference between models $A(E)$ and $\mathrm{B}$ is the initial magnetic field strength. The initial magnetic field energy in Run A with $\beta_{0}=10^{4}$, is 4 orders of magnitude higher than that in Run B. In model E, $\beta_{0}=200$, the initial magnetic energy in this model is 6 orders of magnitude higher than that in Run B.

Figure 6 shows $\log \alpha$ in Run A. In the region $12^{\circ} \leq \theta \leq 168^{\circ}$, $\alpha<\phi$, anisotropic viscosity is much larger than the Maxwell stress. In regions $\theta \leq 12^{\circ}$ and $\theta \geq 168^{\circ}$, Maxwell stress is much larger than anisotropic viscosity.

The mass accretion rate of Run A is shown in Fig. 2. Both mass inflow and outflow rates decrease towards the black hole. In order to investigate why mass inflow rate decreases inwards, we plot Fig. 7. This Figure shows the velocity vectors for Run A. It is clear that in this model, real outflow (wind) is much stronger than that in Run $\mathrm{B}$. Winds dominate in the region $0^{\circ} \leq \theta \leq 24^{\circ}$, in the region $24^{\circ} \leq \theta \leq 45^{\circ}$, there is a turbulent eddy, and in the region $45^{\circ} \leq \theta \leq 90^{\circ}$, turbulent motions dominate.

In order to precisely study the mass flux of wind, we plot the trajectories of gas particles in Fig. 8. From this Figure, we can see that wind dominates in the region $\theta \leq 24^{\circ}$ which is consistent with that found in Fig. 7. Our quantitative calculation shows that the mass flux of wind is $15 \%$ of the mass outflow rate calculated by Eq. (8). Winds are present in the region $\theta \leq 24^{\circ}$. In this region, the gas density is very small; therefore, the mass flux of wind is still small. The mass outflow rate calculated by Eq. (8) is dominated by turbulent motions. Therefore, as in models B

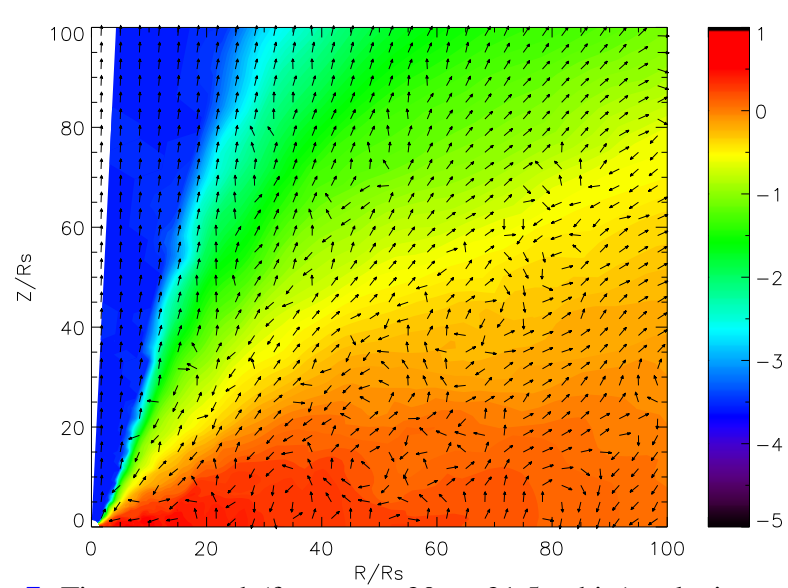

Fig. 7. Time-averaged (from $t=20$ to 21.5 orbits) velocity vectors (arrows) over-plotted by density (colors) for Run A.

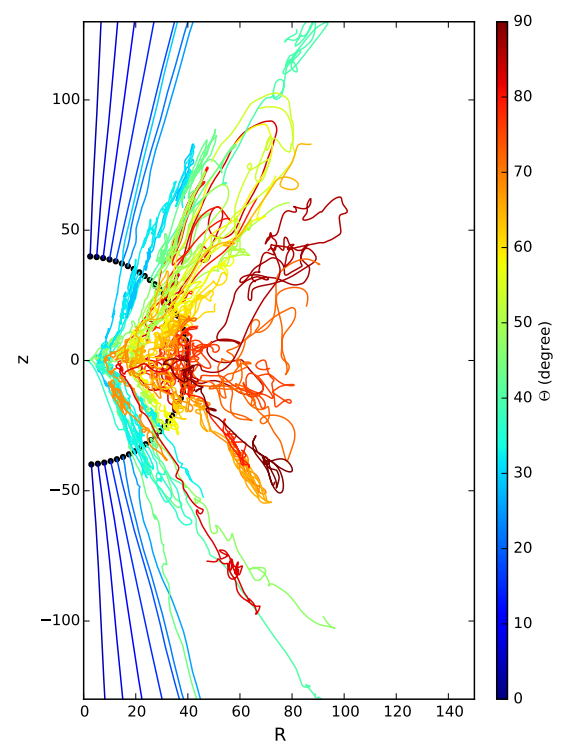

Fig. 8. Same as Fig. 4, but for Run A.

and $\mathrm{C}$, turbulent motions induced by convection cause the mass inflow rate of model A to decrease inward.

For comparison, we also present results from a control run E. In this model the Maxwell stress is comparable to the anisotropic viscosity. We also use the trajectory method to study whether or not winds exist. Figure 9 shows the trajectories of 88 "test particles" starting from $40 R_{\mathrm{S}}$ in model E. It is clear that strong wind exists in the region $0^{\circ}<\theta<45^{\circ}$ and $135^{\circ}<\theta<180^{\circ}$. Our quantitative calculation shows that the mass flux of real outflow (wind) is about $70 \%$ of the total outflow rate calculated by Eq. (8). Wind is very strong in run E. This is consistent with previous numerical simulations (Yuan et al. 2015), which studied hot accretion flow with large Maxwell stress.

\section{Summary and discussion}

In hot, dilute plasmas, the mean-free path of ions can be large compared to the typical scalelength of accretion flow. When the ion mean-free path is larger than its gyroradius, pressure is anisotropic with respect to magnetic field lines. The effects of pressure anisotropy can be modeled by anisotropic viscosity. In non-relativistic limit, the anisotropic viscous stress is reduced to Braginskii viscosity (Braginskii 1965). Angular momentum of the accretion flow can be transferred by anisotropic viscosity. 
A\&A 608, A114 (2017)

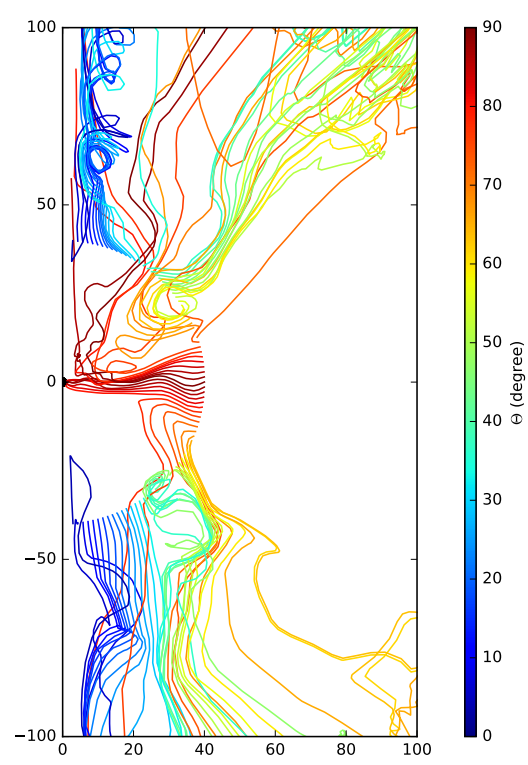

Fig. 9. Same as Fig. 4, but for Run E. Our quantitative calculation shows that the mass flux of real outflow (wind) is about $70 \%$ of the total outflow rate calculated by Eq. (8). Wind is very strong in run E.

In this work, we perform two-dimensional global MHD simulations of hot accretion flow. We investigate hot accretion flow in which anisotropic viscous stress is stronger than Maxwell stress. We find that the flow is convectively unstable. We also find that the mass inflow rate decreases towards the black hole. Wind is very weak; its mass flux is $10-15 \%$ of the mass inflow rate. The inward decrease of inflow rate is mainly due to convective motions. This result may be useful to understand the accretion flow in the Galactic Center Sgr A* and the accretion flow in the galaxy M 87 (Akiyama et al. 2015).

Many observations show that wind is highly prevalent in AGNs and black hole X-ray binaries. The Blandford \& Payne (1982) mechanism, which needs large-scale open magnetic field lines, is usually invoked to account for the origin of wind. But, it is still unclear whether or not large scale magnetic fields exist in accretion flows (Beckwith et al. 2009). Recently, Yuan et al. (2012b) introduced a micro-Blandford \& Payne mechanism to explain the origin of wind without large-scale open magnetic fields. We extend previous work to cases with very weak magnetic field. Our results show that even with extremely weak magnetic field, wind can be present in the accretion flow with anisotropic viscosity.

Acknowledgements. This work was supported in part by the Natural Science Foundation of China (grants U1431228, 11133005, 11233003, 11421303), the National Basic Research Program of China (2012CB821801), the Strategic Priority Research Program of the Chinese Academy of Sciences (XDB09000000), and the grant from the Fundamental Research Funds for the Central Universities. De-Fu Bu and Zhao-Ming Gan are supported in part by the Natural Science Foundation of China (grants 11133005 and 11573051, 11633006), the National Basic Research Program of China (973 Program, grant 2014CB845800), the Key Research Program of Frontier Sciences of CAS (No. QYZDJ-SSW-SYS008), the Strategic Priority Research Program "The Emergence of Cosmological Structures" of CAS (grant XDB09000000), the grant from the Ministry of Science and Technology of China (No. 2016YFA0400704), the CAS/SAFEA International Partnership Program for Creative Research Teams and the Natural Science Foundation of Shanghai (grant 16ZR1442200). This work made use of the High Performance Computing
Resource in the Core Facility for Advanced Research Computing at Shanghai Astronomical Observatory.

\section{References}

Abramowicz, M. A., Chen, X., Kato, S., Lasota, J. P., \& Regev, O. 1995, ApJ, 438, L37

Akiyama, K., Lu, R.-S., Fish, V. L., et al. 2015, ApJ, 807, 150

Balbus, S. A. 2004, ApJ, 616, 857

Balbus, S. A., \& Hawley, J. F. 1991, ApJ, 376, 214

Balbus, S. A., \& Hawley, J. F. 1998, Rev. Mod. Phys., 70,

Beckwith, K., \& Hawley, J. F. 2008, ApJ, 678, 1180

Beckwith, K., Hawley, J., \& Krolik, J. H. 2009, ApJ, 707, 428

Begelman, M. C. 2012, MNRAS, 420, 2912

Begelman, M. C., \& Meier, D. L. 1982, ApJ, 253, 873

Blandford, R., \& Payne, D. G. 1982, MNRAS, 199, 883

Braginskii, S. I. 1965, Rev. Plasma Phys., 1, 205

Bu, D. F., Yuan, F., \& Stone, J. M. 2011, MNRAS, 413, 2808

Bu, D. F., Yuan, F., Gan, Z. M., \& Yang, X. H. 2016, ApJ, 818, 83

Chandra, M., Gammie, C. F., Foucart, F., \& Quataert, E. 2015, ApJ, 810, 162

Foucart, F., Chandra, M., Gammie, C. F., \& Quataert, E. 2016, MNRAS, 456, 1332

Gu, W. M. 2015, ApJ, 799, 71

Hawley, J. F., \& Balbus, S. A. 2002, ApJ, 573, 738

Hellinger, P., \& Trávníček, P. M. 2015, J. Plasma Phys., 81, 013003

Hellinger, P., Trávníček, P. M., Kasper, J. C., \& Lazarus, A. J. 2006, Geophys. Res. Lett., 33, 9101

Igumenshchev, I. V., \& Abramowicz, M. A. 1999, MNRAS, 303, 309

Igumenshchev, I. V., \& Abramowicz, M. A. 2000, ApJS, 130, 463

Islam, T. 2014, ApJ, 787, 53

Islam, T., \& Balbus, S. 2005, ApJ, 633, 328

Johnson, B. M., \& Quataert, E. 2007, ApJ, 660, 1273

Kasper, J. C., Lazarus, A. J., \& Gary, S. P. 2002, Geophys. Res. Lett., 29, 20

Kulsrud, R. M. 2004, Plasma Physics for Astrophysics (Princeton NJ: Princeton Univ. Press)

Kunz, M. W., Schekochihin, A. A., \& Stone J. M. 2014, Phys. Rev. Lett., 112, 205003

Li, J., Ostriker, J., \& Sunyaev, R. 2013, ApJ, 767, 105

Machida, M., Matsumoto, R., \& Mineshige, S. 2001, PASJ, 53, L1

Mahadevan, R., \& Quataert, E. 1997, ApJ, 490, 605

Narayan, R., \& Yi, I. 1994, ApJ, 428, L13

Narayan, R., \& Yi, I. 1995, ApJ, 444, 231

Narayan, R., Igumenshchev, I. V., \& Abramowicz, M. A. 2000, ApJ, 539, 798

Narayan, R., Sadowski, A., Penna, R. F., \& Kulkarni, A. K. 2012, MNRAS, 426, 3241

Ostriker, J. P., Choi, E., Ciotti, L., Novak, G. S., \& Proga, D. 2010, ApJ, 722, 642

Paczyński, B., \& Wiita, J. 1980, A\&A, 88, 23

Pang, B., Pen, U. L., Matzner, C. D., Green, S. R., \& Liebendorfer, M. 2011, MNRAS, 415, 1228

Parrish, I. J., \& Stone, J. M. 2005, ApJ, 633, 334

Parrish, I. J., \& Stone, J. M. 2007, ApJ, 664, 135

Quataert, E. 2008, ApJ, 673, 758

Quataert, E., \& Gruzinov, A. 2000, ApJ, 539, 809

Quataert, E., Dorland, W., \& Hammett, G. W. 2002, ApJ, 577, 524

Riquelme, M. A., Quataert, E., Verscharen, D. 2015, ApJ, 800, 27

Sadowski, A., Lasota, J. P., Abramowicz, M. A., \& Narayan, R. 2016, MNRAS, 456, 3915

Sharma, P., Hammett, G. W., \& Quataert, E. 2003, ApJ, 596, 1121

Sharma, P., Quataert, E., \& Stone, J. M. 2008, MNRAS, 389, 1815

Sironi, L., \& Narayan, R. 2015, ApJ, 800, 88

Stone, J. M., \& Norman, M. L. 1992a, ApJS, 80, 753

Stone, J. M., \& Norman, M. L. 1992b, ApJS, 80, 791

Stone, J. M., \& Pringle, J. E. 2001, MNRAS, 322, 461

Stone, J. M., Norman, M. L., \& Begelman, B. C. 1999, MNRAS, 310, 1002

Tanaka, T., \& Menou, K. 2006, ApJ, 649, 345

Tassoul, J. L. 1978, Theory of Rotating Stars (Princeton, NJ: Princeton Univ. Press)

Wang, Q. D., Nowak, M. A., Markoff, S. B., et al. 2013, Science, 341, 981

Yuan, F., \& Narayan, R. 2014, ARA\&A, 52, 529

Yuan, F., Bu, D. F., \& Wu, M. C. 2012a, ApJ, 761, 130

Yuan, F., Wu, M. C., \& Bu, D. F. 2012b, ApJ, 761, 129

Yuan, F., Gan, Z. M., Narayan, R., et al. 2015, ApJ, 804, 101 


\section{Appendix A: Artificial resistivity}

The Zeus code has issues with losing energy from the grid. In the MHD simulation, there will be numerical dissipation in magnetic field. In order to convert the magnetic energy dissipated in current sheet into thermal heating, we include explicit artificial resistivity in our Eqs. (3) and (4). Following Stone \& Pringle (2001), the coefficient of artificial resistivity is given by:

$\eta=\frac{Q(\Delta x)^{2}}{\sqrt{\rho}}|\boldsymbol{J}|$

Here, $\Delta x$ is the grid spacing, $\rho$ is gas density, $\boldsymbol{J}$ is current density, and $Q$ is a constant.

In Stone \& Pringle (2001), the authors have done some tests. They find that for $Q=0$ the change in the total energy in the flow is $0.6 \%$. If $Q=0.1$ this change is reduced to $0.08 \%$. They conclude that with an artificial resistivity, conservation of total energy is improved because energy losses due to numerical reconnection are reduced. In this paper, we set $Q=0.1$. In Fig. A.1, we plot the time evolution of total energy and its individual components (internal, kinetic, gravitational, and magnetic energies) for model B. It is clear that the total energy (solid line) is almost constant. Also, the magnetic energy is several orders

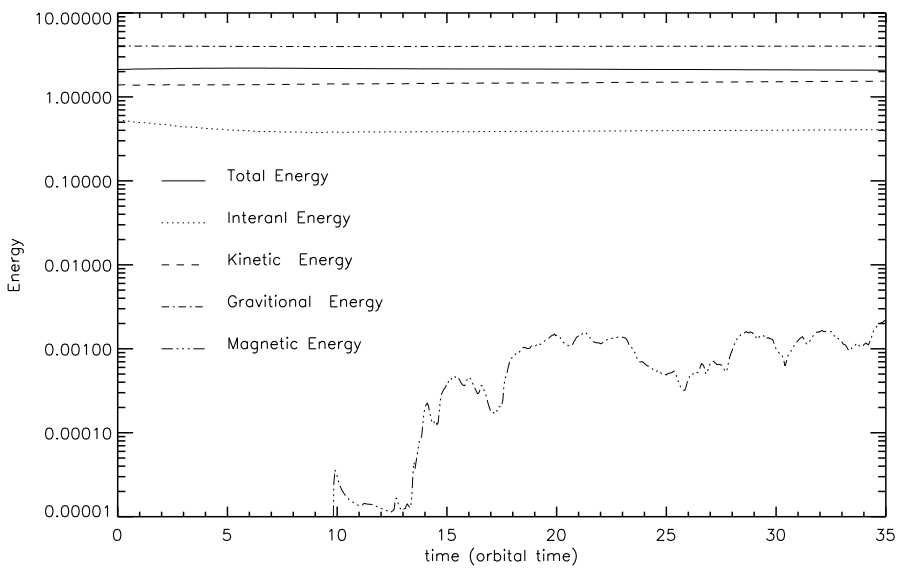

Fig. A.1. Time evolution of total energy and its individual components (internal, kinetic, gravitational and magnetic energies) for model B.

of magnitude smaller than other components of energy. The effect of numerical magnetic energy loss on the total energy evolution of the accretion flow is negligible. 